\title{
CARACTERIZACIÓN CURRICULAR, PEDAGÓGICA Y EVALUATIVA DE LA FORMACIÓN TÉCNICA PROFESIONAL Y TECNOLÓGICA EN COLOMBIA.
}

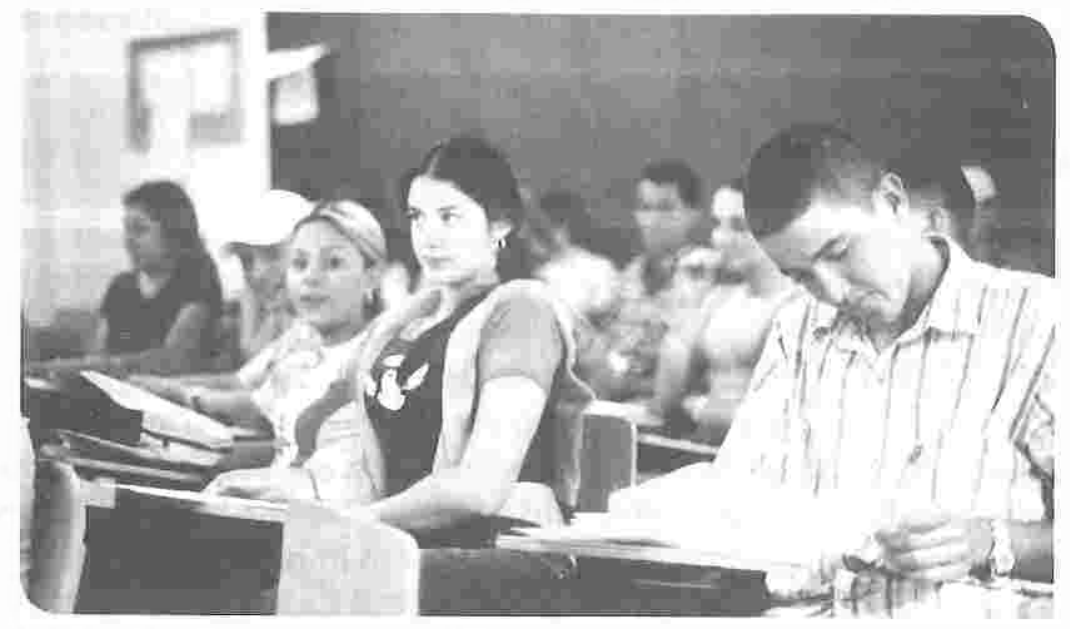

Hernán M. Chaves A.42 Economista, Especialista en Finanzas, Magister en Educación, Rector Corporación Tecnológica de Bogotá, Bogotá, Colombia.

\section{RESUMEN}

El presente artículo, es el resultado de una investigación sobre educación Técnica Profesional y Tecnológica que abordó la problemática existente en relación con la discriminación que se le hace aduciendo que es una educación de baja calidad, que es ofrecida a personas de menores recursos y queen su desarrollo no se realiza investigación ni proyección social, lo que permitió avanzar en la elaboración de planteamientos que facilitaran la comprensión de lo que es la Educación Técnica Profesional y Tecnológica, identificando las posibles soluciones a los problemas detectados propiciando procesos de toma de decisiones y formulación de políticas para el mejoramiento de este nivel de la educación superior. Se revisó el fundamento conceptual y teórico partiendo de los antecedentes de la educación, especialmente lo relacionado con la conceptualización de la Educación Técnica Profesional yTecnológica, sus diferencias y sus espacios comunes, para lograr al final de dicha investigación presentar una

42 Maestria en Educación. Universidad Surcolombiana 
propuesta alternativa para la formación en la Educación Técnica Profesionai y Tecnológica acorde a ios resultados obtenidos en el trabajo de campo. Pese a la compiejidad de la problemática abordada, dicha investigación pretendió encontrar posibles soiuciones a ia misma, permitiendo desarrollar una concepción ciara, argumentada y pertinente de la formación desarroilada por las instituciones de educación superior comprometidas con ia formación Técnica Profesiona! y Tecnológica de Coiombia.

\section{SUMMARY}

This article is the result of an investigation on Technicai Vocationai and Technoiogical Education which addressed the problems in relation to discrimination that is made on the grounds that it is a iow quaiity education, which is offered to people with fewer resources and in its development is not done research or outreach. This perception ied to progress in deveioping approaches to faciiitate the understanding of what the Technicai Vocational and Technoiogicai Education, identifying possibie solutions to the problems identified in order to faciiitate decision-making processes and policy to improve this ievei of higher education. Revised conceptuai and theoretical foundation based on the history of education, especially related to the conceptuaiization of Technical Education Vocational and Technoiogical, their differences and their common areas. Shown regulations reiated to higher education and technicai education and Technoiogy, the current situation of the Technical Vocationai and Technological Education, the conceptualization of the curriculum, teaching practices and assessment as formative aspect to achieve at the end of such research present an aiternative proposal for training in Technical Education Vocationai and Technological according to the results of the fieid work. Despite the complexity of the issues at stake, this research aimed to find possible soiutions to it, allowing to deveiop a clear conception, argued and relevant training deveioped by the higher education institutions committed to technical education and Technology of Colombia.

\section{INTRODUCCIÓN}

La educación superior y concretamente la formación Técnica Profesional y Tecnológica, debe apoyar la consoiidación de un modelo educativo en donde prime ia democracia, que este acorde a los principios de libertad, tolerancia, fraternidad, que sea inciuyente, basado en igualdad de oportunidades y con la posibilidad de constituir una economía con mayor nivel de bienestar sociai, una sociedad más igualitaria y solidaria, de ciudadanos libres y responsables y un estado ai servicio de ios ciudadanos. 
El mejoramiento de la calidad educativa en Colombia, busca el crecimiento económico, el desarrollo social, el bienestar ciudadano, en donde la educación es considerada los rieles sobre los que se avanzarán en dicho crecimiento, por lo cual la política educativa debe encaminar sus acciones hacia el logro de una mayor calidad en todos los niveles educativos, una atención integral a la primer infancia, la busca del acceso y permanencia en la educación, básiçamente en las diferentes regiones nacionales, una pertinencia de la innovación y una gestión trasparente en donde el sector educativo sea ejemplo de buen gobierno.

Se busca una educación acorde con la realidad nacional, en donde la formación sea la demandada por la sociedad, las empresas y el sector público, razón por la cual se propone fortalecer la educación Técnica Profesional yTecnológica con miras a lograr una mayor demanda por este tipo de educación superior.

En este orden de ideas es necesario conocer las características, diferencias y espacios comunes entre la Formación Técnica Profesional y la Tecnológica.

\section{¿Qué es Formación Técnica Profesional?}

La técnica comprende el conocimiento de los procedimientos y el manejo de las habilidades para la fabricación de bienes o la provisión de servicios, orientada al desarrollo de competencias generales, laborales y ciudadanas.

Para la formación Técnica Profesional se deben tener claros los siguientes aspectos: el nivel de formación requerido, el perfil profesional, el campo de desempeño específico (perfil ocupacional), el desarrolio de competencias de cierto tipo y nivel de complejidad. De igual manera siendo consecuentes con lo planteado por la Ley 749 de 2002, por medio de la cual se organiza el servicio público de la educación superior en las modalidades de formación Ténica Profesional y Tecnológica, se puede definir a la Educación Técnica Profesional como la generación de competencias y desarrollo intelectual de aptitudes, habilidades y destrezas al impartir conocimientos técnicos necesarios para el desempeño laboral en una actividad, en áreas específicas de los sectores productivo y de servicios. Comprende tareas relacionadas con actividades técnicas que pueden realizarse autónomamente, habilitando para comportar responsabilidades de programación y coordinación.

\section{¿Qué es Formación Tecnológica?}

La educación Tecnológica abarca un marco mucho más amplio en su tarea de resolución de problemas. 
La educación Tecnológica busca el avance de competencias generales que permitan lograr una crítica reflexiva de adaptación entre el ciudadano y el mundo tecnológico, donde el alumno desarrolla además su propia capacidad para solucionar determinados problemas del área de conocimiento.

De acuerdo a lo planteado por la Ley 749 de julio 19 2002, la formación Tecnológica establece una formación básica común, que se fundamenta y apropia de los conocimientos científicos y la comprensión teórica para la formación de un pensamiento innovador e inteligente, con capacidad de diseñar, construir, ejecutar, controlar, transformar y operar los medios y procesos que han de favorecer la acción del hombre en la solución de problemas que demandan sectores productivos y de servicios del país. De igual, manera comprende el desarrollo de responsabilidades de concepción, dirección y gestión de conformidad a la especificidad del programa respectivo.

\section{Diferencia entre programas Técnico-Profesionales y programas Tecnoló- gicos}

En términos generales, se puede establecer que la educación Técnica Profesional y Tecnológica corresponde a dos niveles de formación de grado en educación superior que conducen a titulaciones que reconocen competencias profesionales, permiten ajustar los tiempos de estudio y trabajo a las necesidades del estudiante, ya que con las competencias adquiridas y la titulación obtenida se le abrirán otras puertas laborales y se le posibilitará seguir creciendo en su proyecto de vida académico.

La técnica comprende el cómo hacer, mientras que la tecnología incluye también el por qué, el para qué, el dónde y el cuándo se produce un determinado producto tecnológico. Ambos tipos de formación pueden sacar provecho de utilizar situaciones problemáticas como contexto para las capacidades que busca desarrollar.

\section{Una mirada crítica a la formación académica del Técnico Profesional y del Tecnólogo en Colombia}

Las instituciones de educación superior enfrentan una doble responsabilidad, por un lado, se les asigna históricamente la función de mantener y velar por la memoria histórica de las sociedades y por otro se les exige innovación, cambio, descubrimiento. En este dilema, es donde se constituye o estructura el proceso de formación profesional. 
¿Qué se entiende por formación?

Desde el punto de vista académico, se entiende por formación a un conjunto de técnicas, métodos y operaciones de enseñanza que facilitan la transmisión dei conocimiento, de las habilidades, destrezas y competencias que debe poseer el egresado de una disciplina o profesión (López Nelson 2001).

En cuanto a la formación el campo de conocimientos es la integración de los saberes específicos de una disciplina y los saberes relacionados con lo pedagógico, investigativo, social, humanista como tai, los cuales deben estar perfectamente interrelacionados para lograr la transmisión efectiva del conocimiento.

\section{Sobre las tensiones curriculares}

El currículo, entendido como la columna vertebral dei proceso educativo, debe ser construido de manera colaborativa en donde la pertenencia social sea el piiar fundamental, lo que impiica que se debe llevar a cabo un cambio de paradigma por parte de los docentes, de manera tai que desarrollen su proceso pedagógico de una manera participativa y con una permanente evaluación dei mismo, con miras a formar el nuevo ser humano que ei país necesita para lograr superar ias debiiidades del presente. Es decir se debe estructurar un currículo en concordancia con las características sociocultur ales y económicas de cada región o país (Hoyos Santander et al. 2009).

Acorde a lo establecido por ia Ley 115 de 1994 , en su artículo 76 , se puede establecer como currícuio al conjunto de criterios, planes de estudio, programas, metodoiogías y procesos que contribuyen a la formación integral y a la construcción de la identidad cultural, regional y iocal, inciuyendo también los recursos humanos, académicos y físicos para poner en práctica las políticas y lievar a cabo ei proyecto educativo institucional.

\section{Acerca de la problemática pedagógica}

Ei proceso pedagógico es parte sustantiva de la acción formadora que comporta la educación, no obstante, ía pedagogía se convierte en un campo en don de su abordaje en ocasiones ia concibe como técnica, como procedimiento, como disciplina, como arte, como ciencia, como dispositivo. La riqueza de la multiplicidad de formas de abordar la pedagogía deja entrever que es un campo en construcción, asumiéndose la pedagogia como un proceso de interacción sociai que persigue provocar cambios en los sujetos que permitan su adecuación a formas específicas de los contextos en los cuales se desenvueive. 
La educación debe ser el vehículo que permita desarrollar las habilidades del individuo, que lo convierta en un ser integral, responsable, autónomo, que afecta positiva o negativamente el espacio en que se desenvuelve, pero sobre todo que debe respetar a los demás protagonistas de su entorno. De igual manera, la educación se concibe como un proceso continuo, de construcción, de deconstrucción y de reconstrucción de todas las experiencias del ser humano, ya que articula los intereses sociales, culturales y familiares del estudiante, así como la superación de las experiencias tradicionales de la repetición y del aprendizaje memorístico buscando la creatividad y la innovación, en donde las necesidades de la sociedad son tenidas en cuenta de manera fundamental.

\section{Acerca de la problemática de la evaluación}

En los últimos años se han presentado muchos cambios en las estructuras de las sociedades, razón por la cual los logros esperados en educación, definen los aspectos que los estudiantes deben aprender y ser capaces de ejecutar, de acuerdo a sus actividades académicas; definen qué desempeño se aceptará de acuerdo al resultado del aprendizaje que las instituciones de educación les han inculcado, mostrando la misión institucional e indicado el camino para docentes, estudiantes, padres de familia y administradores que debe seguirse para lograr desarrollar las competencias en los diferentes niveles del sistema educacional.

Es importante tener en cuenta que los logros esperados también deben reflejar los valores, actitudes, la formación del carácter de los estudiantes, el patriotismo, la tolerancia, la no violencia y el respeto por los derechos humanos y la vida. Así mismo, la evaluación debe ser formativa y progresiva, en donde los procesos que se definan y las actividades a desarrollar deben ser el centro de la misma, lo cual implica una total compenetración entre el estudiante y el docente, a fin lograr romper las barreras que les impiden observar las capacidades, cualidades y ventajas conjuntas, facilitándoles el cumplimiento de las metas establecidas inicialmente.

En consecuencia, la evaluación puede analizar se desde dos puntos de vista. El primero que asume la evaluación como una acumulación de conocimientos y habilidades y como producto terminal de un proceso de enseñanza. El segundo manifiesta la necesidad de la participación de todos los involucrados en el proceso de enseñanza aprendizaje centrado en el pensamiento y el significado, razón por la cual, la necesidad de presentar y secuenciar la información, organizar las practicas, buscar retroalimentación, integrar las actividades prácticas con las teóricas, entre otras, son el proceso fundamental de la evaluación. 
En general se puede establecer que la evaluación recoge información, la cual es analizada a fin de presentar alternativas de me jor amiento mediante la toma de decisiones. A su vez, es un componente fundamental del proceso formativo, ya que debe responder preguntas como: ¿Qué se enseña?; ¿Cómo se enseña?; ¿Quién aprende?; ¿Cómo aprende?;¿Qué se evalúa?; ¿Cómo se evalúa?¡¿Para qué se evalúa?, todo ello con el propósito de reconocer en el estudiante un individuo integral en donde se indaga sobre lo enseñado, así como, lo considerado por el receptor principa! de la acción enseñanza aprendizaje.

\section{RESULTADOS Y DISCUSIÓN}

A partir de los análisis, la tendencia que se impone en relación con el concepto de formación, es la que asocia a la formación con los contenidos, métodos y modelos que permiten la construcción de competencias en el futuro profesional, permitiéndole realizar una actividad con eficiencia y eficacia, asi mismo, es la posibilidad de transformar al individuo desde la dimensión del saber, del saber hacer y del ser, en coherencia con el entorno en el cual se desenvuelve, dando una respuesta positiva a las necesidades de la sociedad.

Así mismo, la formación se concibe como un proceso de preparación del sujeto para la vida en todos los órdenes. Obedece a una concepción del hombre desde las dimensiones biológica, cognitiva y afectiva, que determina una ruta formativa con múltiples intereses yen diversos escenarios. La formación se encuentra asociada a una indagación permanente, en donde el docente, el estudiante, la familia, la institución, entre otros, genera una posición diferente de cada uno de estos en el papel que desempeñan en el proceso de enseñanza aprendizaje, a fin de lograr una adecuada articulación entre un campo de problemas y un cam po de conocimientos para desarrollar su programa açadémico con pertenencia social y pertinencia académica.

Lo anterior implica una estrecha relación entre la formación y las estructuras curriculares, las prácticas pedagógicas y los procesos evaluativos

La Formación Técnica Profesional prepara al estudiante para el hacer, enfocada básicamente en la práctica, desenvolviéndose en niveles operativos, rutinarios y poco comple jos. En este tipo de formación se desarrollan competencias orientadas a ejecutar actividades laborales con un alto componente especifico, $_{r}$ es decir, el egresado adquiere un conjunto de aptitudes, habilidades y destrezas para desarrollar actividades en áreas específicas.

La Formación Tecnológica desarrolla el saber y el hacer fundamentado en el saber científico, permitiendo una mayor capacidad de gestión, decisión y pro posición de mejoras en los lugares en donde se desempeña el egresado de 
este nivel de formación, dándole una mayor capacidad de gestión, decisión y proposición para mejorar los sistemas donde ejecuta sus actividades laborales.

En cuanto a la investigación, esta es consider ada como una sola, dependiendo su aplicabilidad, del nivel de complejidad y del tipo de formación. Es decir, en la formación Técrica Profesional los procesos de investigación se concentran en la sistematización y anális is de las prácticas laborales asociadas al área profesional con el fin de identificar oportunidades y alternativas de mejora. Busca una solución concreta a problemas específicos del saber técnico de los procesos productivos, preparando al estudiante en una cultura científica básica, obteniendo información relacionada con dicha solución, organizándola, clasificándola, interpretándola encontrando soluciones a problemas de manejo operativo.

En la formación Tecnológica, los procesos de investigación están relacionados con la aplicación de innovación y creatividad. Encuentra soluciones fundamentadas en la ciencia para reflexionar las relaciones causa efecto de los temas para estar en capacidad de crear, transformar, cambiar, mejorar, controlar y ordenar la realidad para la implementación de nuevas tecnologías de producción.

En cuanto a lo curricular, los diseños curriculares están basados en la flexibilidad como un proyecto orientado a hacer viable la formación integral del estudiante a partir de la vivencia de experiencias educativas con los retos de la realidad y con las exigencias intelectuales y sociales de los respectivos campos disciplinares y prof esionales.

Los currículos articulan acciones de docencia, investigación, proyección social y bienestar, con miras a lograr unos mejores resultados en el proceso de en señanza-aprendizaje, pero también como una estrategia pedagógica desde las ciencias, las disciplinas y las profesiones mediante la transformación de las prácticas profesionales y disciplinares que permitan un acompañamiento al estudiante en la construcción del conocimiento, a través del aprendizaje autónomo, la interrelación entre el estudiante y el docente en donde el currículo se interpreta como un campo de investigación y de prắctica pedagógica permanente.

Se establece el currículo desde un contexto académico disciplinar en donde la pertinencia académica es el factor fundamental, ya gue establece las competencias como un saber hacer en contexto, dándole el sentido a la formación disciplinar buscada por la institución y desde su pertinencia social, cultural y económica en donde lo que se enseña tiene un aplicabilidad en las necesidades del contexto. 
El currículo está enmarcado en el modelo pedagógico de las instituciones de educación superior como el contenido temático que habilita al estudiante mediante la apropiación de herramientas para que este pueda aprender a hacer, a mane jar campos de conocimiento investigativos, de proyección social, de la ciencia y de la tecnología para el desarrollo del conocimiento.

A pesar que el currículo es entendido como un conjunto de objetivos, contenidos, criterios metodológicos y de evaluación que orientan la actividad académica, aún existe una fuerte tendencia en entender éste como el plan de estudios.

En lo pedagógico, se evidencio que el modelo pedagógico prevaleciente está orientado a la búsqueda del cambio cualitativo del estudiante, mediante el logro de nuevas formas de ser, de hacer con conciencia y de orientar las diferentes disciplinas estructuradas en las instituciones, trascendiendo el esquema educativo tradicional hacia una adecuada practica pedagógica desde la interacción con el conocimiento y el entorno social para lograr una adecuada apropiación del saber, de la relación entre los sujetos y el saber y de las condiciones necesarias que el docente debe apropiar para logra un adecuado aprendizaje en el estudiante. Así mismo, se evidencio que el docente apropia su papel de formador, mediante la capacidad para dialogar su saber con colegas, desarrollar la investigación como factor fundamental de su disciplina, apropiar un compromiso ético consigo mismo, con el conocimiento, con la cultura, pero fundamentalmente con el estudiante, posibilitándole la construcción de competencias, asegurando una educación de cal idad, tanto a nivel local, regional como nacional.

El modelo pedagógico y los procesos de aprendizaje, son construidos desde la pregunta yen el debate que cuestiona, ya que genera reflexiones sobre los fundamentos de las ciencias y los campos de aplicación. Promueve el trabajo autónomo del estudiante desde el compromiso del docente como facilitador, tutor, indagando, descubriendo, resolviendo los problemas de conocimientos y de la vida práctica, promoviendo iniciativas e impulsándolas a nuevos escenarios investigativos a partir de su formación disciplinar y profesional, todo esto enmarcado en los principios de autonomía, libertad de enseñanza, de aprendizaje y de investigación, garantizando la libertad de cátedra como la discrecionalidad que tiene el docente para exponer los fundamentos de su disciplina con criterio ético, científico y permanente actitud investigativa, promoviendo la cultura del diálogo, la participación, el respeto y las buenas relaciones en la diversidad de pensamiento ode iniciativa. 
A pesar de lo anterior, aun es una realidad que muchos modelos pedagógicos de las Instituciones de Educación Superior desarrollan prácticas pedagógicas basadas en la trasmisión de conocimientos, la memorización de estos y su repetición en el momento de las evaluaciones, evidenciando dichos modelos centrados en la actuación, con representaciones fuertemente encaminadas hacia la reproducción y consolidación de lo existente.

Las instituciones de educación superior han modificados sus modelos pedagógicos hacia el aprendiza je del estudiante de jando de lado la enseñanza del docente, sin embargo, el proceso formativo continua siendo repetitivo, academicista, transmisionista y descotextualizado, centrado en una actuación debidamente definida, quien transmite (el docente), lo que se transmite (el discurso) y a guien se transmite (el estudiante), con estructuras de poder y control claramente definidas, evidenciándose el mantenimiento de lo conocido y lo tradicional. Así mismo, se entiende como práctica pedagógica el proceso mediante el cual se afecta la conciencia del otro con el propósito de transmitir, reproducir y transformar la cultura.

Lo pedagógico no es lo didáctico, es decir, lo gue se hace en el escenario del salón de clase, por el contrario, se puede afirmar que lo pedagógico es el resultado de una interacción social que desborda el ámbito escolar, por eso es la relación que debe darse en la interacción profesor alumno, en la cual se plantean tres componentes básicos: 1. Cómo el docente enseña, es decir, cómo es su capacidad para enseñar; 2 Cuál es la motivación gue el estudiante recibe del docente para aprender, ya gue de esta forma el estudiante estará seguro que aprende para entender y aplicar a futuro el conocimiento adguirido; 3 . Cómo se desarrolla la actividad de enseñanza aprendizaje como tal, ya que si esta no entretiene al estudiante, este se aburrirá, desmotivará y por lo tanto su aprendizaje no será el mejor posible.

En lo evaluativo, la evaluación para muchas instituciones de educación superior es un proceso eguitativo gue le facilita una mejora continua en el proceso de enseñanza aprendizaje al estudiante, brindándole las herramientas necesarias que le ayuden en su proceso de formación y avanzar en su programa académico. De igual forma, la evaluación es considerada como una herramienta que valora el ejercicio docente, permitiéndole a este realizar correctivos en su proceso de enseñanza y de esta forma lograr, de la mejor manera, la transferencia del conocimiento, ya que este debe ser constructor de su propia evaluación, pero en concordancia con los estudiantes. Todo esto facilita que el estudiante crezca en la apropiación de su conocimiento demostrando su fortaleza en la adquisición de elementos que le permita responder por su ser. 
posibilitando al estudiante una mejor comprensión del mundo, sus propios campos profesionales y los problemas y necesidades de la sociedad.

En general, tiene que existir una adecuada relación entre las estructuras curriculares, las prácticas pedagógicas y los criterios de evaluación, aplicados a la Educación Técnica Profesional y Tecnológica, porque si no la institución estaría trabajando desarticuladamente.

Teniendo en cuenta las exigencias normativas del Ministerio de Educación Nacional, para los estudiantes que desarrollan programas Técnicos Profesionales y Tecnológicos, es prioritario facilitarles los fundamentos necesarios para que adquieran una formación integral entre lo práctico y lo teórico.

Es necesario que los procesos de evaluación y autoevaluación de los diferentes programas de formación Técnico Profesional y Tecnológico desarrollados por las instituciones de educación superior estén integrados entre ef sector productivo y el académico, bien sea de manera combinada o bien de forma paralela, a fin de revisar y actualizar los diseños de dichos programas.

La generación y construcción de la evaluación, acorde a lo establecido conjuntamente entre el docente, el estudiante y demás participantes del proceso enseñanza aprendizaje, es un factor fundamental para lograr un adecuado desarrollo del proceso académico.

Cuando no se escucha, atiende, interpreta o acoge la opinión, el querer y la voluntad de los estudiantes y demás grupos vinculados al proceso académico, el acto formativo no funciona adecuadamente y por lo tanto no se logran los objetivos queen la actualidad le corresponde a la educación y espacialmente a la educación superior. Por esta razón es necesario crear espacios y mecanismos de participación en los cuales escuchar a los jóvenes, realizar deliberaciones y debates en tono al proceso educativo afirman la idea de que la educación es un asunto de todos los actores formativos.

Es fundamental la participación de toda la sociedad yen especial de los directivos, docentes, estudiantes y padres de familia para lograr una mayor calidad de la educación, enfatizando en la Formación Técnica Profesional yTecnológica.

De igual forma, es fundamental lograr una mayor responsabilidad y compromiso de los docentes para lograr que los egresados sean lo suficientemente competentes para ingresar al mercado laboral.

Es necesario propender para que la formación Técnica Profesional yTecnológica sea bien aceptada en la sociedad y especialmente en ef mercado laboral, a 
fin de que los actores de estos niveles los valoren de acuerdo a las necesidades de la región en donde se desarrolla este tipo de formación.

Los proyectos de educación superior hoy deben responder tanto a las necesidades de contribuir al desarrollo de la ciencia, la tecnología y las artes, como a las exigencias del sector productivo y a las expectativas de los jóvenes, aportando programas académicos con pertinencia, dando mayor cobertura con calidad, en especial a los grupos menos favorecidos, utilizando herramientas tecnológicas de última generación, formando en competencias a través de metodologías pedagógicas flexibles y currículos determinados por las necesidades y exigencias del sector productivo, facilitando así la inserción temprana del estudiante al medio laboral.

El fortalecimiento de la educación Técnica Profesional y Tecnológica favorecerá también la movilidad de los estudiantes entre los ciclos de formación y en los distintos niveles del sector productivo.

A partir de los elementos o factores a destacar encontrados en desarrollo de la investigación que ha motivado el presente documento, y de acuerdo con los objetivos de la misma, a continuación se plantean los elementos estructurantes de una propuesta de intervención de los procesos de formación Técnico Profesional y Tecnológico, que permiten repensar los val ores y debilidades encontradas.

La propuesta académica (curricular, pedagógica y evaluativa) que se propone responde a las realidades locales, regionales, nacionales y globales, con el fin de lograr el mejoramiento de la calidad de vida de los diferentes seres humanos que son formados o resultado de la educación, así como de las comunidades, las regiones, los países y del mundo, siendo la innovación, la formación en competencias laborales y básicas y la cualificación docente fundamental para alcanzar las metas propuestas por la Educación Superior y fundamentalmente por la Instituciones de Educación Superior con programas en formación Técnica Profesional yTecnológica.

Con el propósito de integrar la pertinencia académica y la pertenencia social con las necesidades que orientan y determinan una educación Técnica Profesional y Tecnológica, es necesario transformar los paradigmas actuales de la educación, buscando una propuesta alternativa que ajuste los currículos, las prácticas pedagógicas y el proceso evaluativo a las nuevas exigencias y retos planteados, siendo las formas y métodos de conocer más importantes que el conocimiento mismo; la relación docente estudiante deja de ser una interacción de autoridad para convertirse en totalmente participativa; la acción docente es integral y flexible y 
la evaluación, entendida como el análisis objetivo, desapasionado y concreto de los aciertos y errores generados antes, en y después del proceso de formación académica (López Nelson 1995), es concertada y acorde a las formas de interacción entre el docente y el estudiante, en donde la formación del docente debe ser integral, ya que esta tiene un impacto significativo, debido a que su saber (la disciplina que socializa), su hacer (la didáctica que utiliza) y su ser (valores éticos), ayudan a enfrentar los cambios acelerados de la época actual, pero sobre todo, influencian el excelente desarrollo de los individuos (estudiantes) sobre los cuales puede extender su proyecto de vida, debido a que los docentes son los agentes de primer orden que orientan los procesos de transformación de las instituciones de educación superior (Fernández Martha et al 2008).

De igual forma, esta transformación implica cambios en la manera de pensar y actuar la educación superior, generando nuevas actividades de interacción entre el hacer, el ser, el saber y el saber hacer en la educación Técnica Profesional yTecnológica, con miras a establecer las relaciones mutuas y los requisitos básicos para desarrollar adecuadamente las alternativas presentadas.

Así mismo, se debe tener en cuenta los aspectos cognitivos, afectivos y fisiológicos de los estudiantes a fin de lograr el total involucramiento de estos en el proceso académico, lo que facilitará la interacción y su respuesta al aprendizaje, pues estos deberán ser ciudadanos del mundo con el adecuado desarrollo de su personalidad, respetuoso de los derechos, deberes, diversidad cultural, étnica, ambiental, que vivan en paz y armonía con sus semejantes y la naturaleza en la que está inmerso. En términos generales que sea autónomo y competente en su desempeño personal, social y laboral (Fernández Martha et al, 2008).

En este orden de ideas, se debe buscar una formación en donde el compromiso social y político sean las variables fundamentales; el dialogo permanente entre los docentes, estudiantes y comunidad en general sea una premisa para crear y fortalecer la formación integral; los programas de formación tengan pertenencia social y pertinencia açadémica; la investigación inter disciplinaria y la innovación factores esenciales para el desarrollo de la sociedad y de la economía y las unidades curriculares estén integradas al propósito y al perfil de formación.

La propuesta alternativa presentada está basada en los avances logrados por el Grupo de Investigación PACA (Programa de Acción Curricular Alternativo), buscando la consolidación de una cultura institucional que permita generar nuevas identidades profesionales e interdisciplinarias, en donde cada una se esté construyendo, de-construyendo y reconstruyendo permanentemente y se conviertan en las "hojas de ruta" de las instituciones de educación superior 
de carácter Técnico Profesional y Tecnológico que acepten el reto de romper los paradigmas existentes, pero gar antizando siempre la per tinencia académica y la pertenencia social.

Se parte de un cambio del paradigma existente, a fin de evolucionar favorablemente hacia las nuevas posibilidades y contextos que se están presentado en la actualidad sobre educación Técnica Profesional y Tecnológica, para finalizar en el desarroilo de un modelo que involucre todos los factores que tienen relevancia en la estructuración de un programa académico en donde la indagación permanente es la esencia del mismo.

En este orden de ideas, este contexto debe observarse desde el punto de vista holistico e integral, (macro), es decir a nivel de tendencias generales de carăcter económico, político, social, cultural, demográfico, jurídico, investigativo, científico y tecnológico en el entorno internacional y nacional buscando una mirada de las expresiones socio culturales generales del espacio en donde se inscribirá el programa académico.

El contexto micro, hace referencia a situaciones especificas de cada institución en la cual se va a implementar el programa académico, identificando las fortalezas y debilidades, enfatizando en las oportunidades y falencias de la institución mostrando los recursos y capacidades con que cuenta. Es decir se deben analizar aspectos tales como los planes y politicas regionales, las tendencias y desarrollo sectorial, los aspectos teleológicos de la institución, los programas of recidos, la capacidad investigativa, la planta física, el mobiliario, las ayudas didácticas, la formación de los docentes, entre otros, en otras palabras se debe enfocar hacia un proceso de detección de las necesidades, permitiéndose una argumentación y justificación de los diferentes programas académicos ofrecidos por cada institución de educación superior.

Una vez detectadas las necesidades reales, es fundamental priorizarlas y jerarquizarlas a fin de garantizar la pertenencia social, es decir, de qué manera la institución responde a necesidades específicas de su área de influencia y la pertinencia académica, es decir, como desde el punto de vista académico los programas responden a las necesidades detectadas, todo ello dentro del proceso curricular adelantado.

Una vez establecida la pertinencia académica (respuesta válida en el campo formativo (disciplinario, interdisciplinarios, investigativo, extensión y proyección social, entre otras) a las diversas necesidades detectadas y la pertenencia social (necesidades de diferente indole que dan origen al Programa), se 
debe desarroliar un trabajo reflexivo, crítico y objetivo conjuntamente entre los equipos de trabajo académico institucional, con la visión de los gremios, asociaciones, comunidades académica, científica, sector productivo, sector internacional, entre otros, para definir las responsabilidades, conocimientos, aptitudes y actitudes necesarias para desarrollar responsablemente el programa académico debidamente definido.

Lo anterior implica que se requiere no solo conocer la actuación profesional ideal del egresado, sino también, la realidad concreta del desempeño, definiéndose de esta manera el propósito de formación, siendo este el resultado de un proceso de participación, cooperación, negociación y concertación creando un clima democrático en donde los participantes pueden expresar libremente su pensamiento. De igual forma el propósito de formación es la descripción de las características fundamentales que identifican y definen la especificidad y peculiaridad del proceso académico que dada su importancia se considera como el norte o la misión de cada uno de los programas educativos.

Definido el propósito de formación, es necesario establecer cómo se va a dar a conocer el mismo, es decir, se debe definir la estrategia o el plan operativo adecuado que garantice la relación entre la actividad práctica del individuo y su actividad teórica, así como el desarrollo de acciones participativas entre los individuos y las diferentes formas de solucionar los problemas propuestos, con el fin único de lograr dicho propósito de formación.

Esta estrategia de formación se denomina núcleos temáticos y problemáticos, entendidos estos como "el conjunto de conocimientos afines que posibiliten definir prácticas y procesos de investigación en torno a un problema común" (López Neison 2001). Lo cual permite integrar un campo de problemas con un campo de conocimientos directamente relacionados con el programa que está en ejecución, facilitando la integración entre las funciones sustantivas de las instituciones de educación superior como son, la docencia, la investigación y la proyección social e incluso poder articularlas con la internacionalización y el bienestar de los estudiantes en las diferentes instituciones educativas.

En este orden de ideas, se puede estabiecer que los núcleos temáticos y problemáticos se convierten en un articulador totalmente contrario a la concepción de asignaturas aisladas y sin coordinación dentro de un plan de estudios, garantizando la relación entre la teoría, la práctica, la participación y el trabajo en equipo para la resolución de los problemas. 
De igual forma, es importante considerar que los núcleos temáticos y problemáticos se determinarán de acuerdo a las necesidades del propósito de formación, por lo cual pueden existir algunos que desarrollen su función de una manera transversal, presentándose en todo el proceso formativo, mientras que otros solamente obedecerán a la soiución de problemas puntuales.

En este orden de ideas, al convertirse los Núcleos Temáticos y Problemáticos en la unidad integradora de los diferentes saberes, une la docencia, la investigación, la proyección social, la internacionalización y ei bienestar institucional como elementos básicos del proceso académico: Integran la practica con la teoría; al usuario beneficiario del proceso educativo con la realidad en donde desarrolla su proceso laboral; a los docentes, ya que cada uno desde de su campo de formación aporta y participa en el desarrollo del proceso académico y a las diferentes asignatura convirtiendo su labor en un curso específico.

Dada la complejidad de los Núcleos Temáticos y Problemáticos, estos pueden dividirse en Bloques programáticos, entendidos estos como estructuras que organizan el manejo y desarrollo de las dimensiones, problemas, objetos y principios que constituyen un núcleo temático y problemático.

Estos bloques programáticos, generan proyectos específicos que dan respuesta a las inquietudes planteadas desde la integración de la docencia, la investigación, la proyección social, la internacionalización y el bienestar institucional.

Como un valor agregado de la investigación desarrollada, se precisa la existencia de una visión alternativa y enriquecida del proceso de formación profesional. Esta se encuentra asociada a procesos de indagación permanentes, su desarrollo genera una concepción diferente de los roles que desempeñan cada uno de los agentes del acto formativo. La formación en esta concepción se entiende como la sintesis creativa entre un campo de conocimientos y un cam po de problemas que generan características determinantes de los procesos a partir de la duda, la incertidumbre y la creación colectiva que su desarrollo comporta.

La Práctica Pedagógica se entiende como un procedimiento mediante el cual se ejerce control en una relación social, incidiendo, de uno u otro modo, en la conciencia del otro. Este procedimiento adquiere diferentes formas, atendiendo los diferentes contextos (la familia, la escuela, el trabajo, entre otros); sin embargo, su propósito siempre deberá ser el mismo: la transmisión, reproducción o transformación de la cultura.

Con el anterior referente se propone a las Instituciones de Educación Superior Técnica Profesional y Tecnológica, avanzar en la consolidación de un proceso 
formativo orientado, entre otras, por las siguientes características o aspectos determinantes: a) Fundamentado en la Pedagogía Dialógica que permite convertir las potencialidades y las capacidades de los estudiantes en competencias (Relación simbiótica entre el saber, el hacer y el desear o querer hacer). b) Desarrollado mediante estrategias cognitivas, potestativas, de ejecución en contextos variados y deseadas o anhelantes para que el estudiante de los Programas de Formación Técnica Profesional y Tecnológica, encuentre y construya sus propias maneras de aprendizaje y organice sus procesos mentales de acuerdo a sus intereses y metas específicas. c) El modelo formativo que se sustenta en las competencias, supera la simple asimilación del conocimiento. Se convierte en una estrategia asociada a la investigación y al cultivo de la capacidad de asombro. Su finalidad está directamente relacionada con la construcción de sentido y con la solución de problemas de toda índole. En este modelo, los roles de los agentes están determinados por la búsqueda de respuestas satisf actorias a preguntas pertinentes. En consecuencia se pueden reseñar los siguientes: ajEs coherente con la dimensión teleológica del Proyecto Educativo institucional; b) Supera la relación asimétrica docente-alumno; c) Los problemas y su solución, se convierten en lo esencial de este modelo; d) El trabajo en equipo, es su estrategia metodológica fundamental; e) El asombro, la incertidumbre y la duda soportan el proceso de formación; f) Su esencia es la investigación permanente; g) Logra en el estudiante propiedades relacionadas con la autonomía, el análisis simbólico, la lectura crítica y propositiva y, la innovación permanente; h) La interdisciplinariedad, la transversalidad y la transdisciplinariedad, se convierten el "ethos" formativo de este mode lo; i) La evaluación se convierte en un proceso académico resultado de la concertación y de los productos alcanzados. Elimina la concepción de evaluar desde el "déficit", y resalta los procesos desarrollados.

Se puede deducir que en el modelo formativo propuesto, la concepción de docencia, investigación y evaluación, recoge la impronta de su estructura, es decir, genera una identidad particular y singular. No es lo mismo formar para etapas iniciales (cognitivas), que formar para la comple jidad (pensamiento crítico y proposicional)

La propuesta anteriormente enunciada implica un cambio en: a) En los principios organizativos del conocimiento, ya que nada más complejo de argumentar que la organización actual del conocimiento, expresada a través de las estructuras curriculares atomizadas y yuxtapuestas. Su énfasis disciplinario no responde a las emergencias de la actual composición y dinámica del conocimiento. La realidad histórica y social (local, regional, nacional e internacional) apenas ejerce una influencia temática y nocional. Son estructuras descontextualizadas, a las cuales se les puede atribuir buena parte de la 
responsabilidad con la deserción estudiantil actual. Un proceso de re-estructuración serio y pertinente tiene que intervenir estas formas organizativas, es decir, tiene que intervenir las estructuras de poder y de control que las soportan. Los Núcleos Temáticos y Problemáticos se constituyen en una alternativa curricular viable para superar la realidad anteriormente aludida; b) En la naturaleza de las relaciones entre los agentes involucrados en los procesos formativos, debido a que la identidad de los actores del proceso formativo debe ser intervenida de manera sustantiva. El trabajo individual debe dar paso al trabajo colegiado; la asimetría presente en la relación profesor-alumno no puede mantenerse incólume e inmodificable. Si la investigación o la indagación sistemática es la esencia del proceso formativo, la corporatividad de estudiantes y profesores frente a los retos del conocimiento, se erige como la estrategia viable y pertinente para "resignificar y recomponer" las relaciones, entre los agentes o actores de la acción formativa. No es posible seguir manteniendo "agendas particulares" si se trata de responsabilidades colectivas; se hace necesario crear estrategias metodológicas para integrar los procesos sustantivos de la Educación Superior (docencia, investigación y proyección sociali), y con ellos, a los agentes, que los realizan; c) En los contextos de interacción ode las prácticas pedagógicas. Lo formativo nose agota en lo escolar; la flexibilidad en todas sus expresiones (personal, académica, pedagógica, curricular, administrativa) asume que el aula de clase, el espacio físico del salón, no es el único y legitimo espacio formativo.

Si las prácticas pedagógicas se consideran procesos de interacción social, no se puede considerar que éstos sólo tengan lugar en la institución como ente formal. Por el contrario, existen otros contextos que deben ser incorporados al proceso formativo para garantizar efectivamente gue se presente una pertinencia efectiva de la acción formativa. Un elemento gue soporta esta afirmación se relaciona con la exigencia oficial de trabajar a partir de créditos académicos.

Si se comparte que el crédito académico, entendido como la unidad de medida del trabajo académico del estudiante, que conjuga la labor realizada con la asistencia del profesor (presencial) y la que adelanta el estudiante de manera independiente, se exige un replanteamiento del proceso formativo, en donde la enseñanza del profesor de paso al aprendiza je del estudiante. Esta adopción o incorporación exige cambios sustantivos y estructurales del "paradigma formativo" vigente o hegemónico en la formación Técnica Profesional y Tecnologica actual.

Es posible que los trabajos que el estudiante realice con la asistencia directa del profesor se celebren en el salón de clase, pero las acciones o trabajos 
independientes del estudiante, deben involucrar, otros espacios formativos, como el hogar, el trabajo, el deporte, la cultura. Analizar seriamente esta necesidad de cambio, debe ser acogida como un acto de responsabilidad yética institucional.

Todo proceso de reforma académica, es el resultado de un acto consciente y argumentado, razón por la cual, como producto concreto de la investigación desarrollada, resulta adecuado enunciar algunas recomendaciones, que deben ser leídas en el contexto de la autonomía institucional, asociada a la dinámica de todas las instituciones responsables de la formación de profesionales en las diversas áreas del conocimiento y dentro del proceso de responsabilitdad social inherentes a cada una de ellas.

En cuanto a recomendaciones que se generan en desarrollo de la investigación, las misma pueden ser derivadas a: a) Las Instituciones: 1- Es necesario asumir los procesos curriculares, pedagógicos y evaluativos como acciones dinámicas, producto o resultado de desarrollos investigativos concretos, muy en correspondencia con la dimensión teleológica que orienta la dinámica de las instituciones Técnicas Profesionales y Tecnológicas. 2- La formación no debe restringirse a un proceso de transmisión de conocimientos o saberes circunscritos a la geografía institucional, por el contrario, en la medida que la formación recoja señales de otras agencias formativas, la familia, el trabajo, la dinámica social, cultural, investigativa, Tecnológica, científica, se convertirá en una estrategia integral y holística, muy coherente con los anhelos de calidad y excelencia que direccionan la dinámica de las Instituciones Técnicas Profesionales y Tecnológicas. b) Los Programas: 1-Es necesario retomar a través de los Comités Curriculares la necesidad de entender que la formación de un profesional a nivel Técnico Profesional y Tecnológico, supera la noción de desarrollo instrumental o procedimental y se instala en un proceso de investigación e indagación sistemática acorde con el propósito de formación y el perfil profesional considerados norte de toda acción formativa. 2- Lo curricular, to pedagógico y lo evaluativo, no son acciones terminales e inmodificables, por el contrario, son resultado de procesos de elaboración permanente y colectiva a los cuales se accede por aproximación sucesiva, razón fundamental para sugerir de manera respetuosa llevar a la practica un proceso de deconstrucción de sus actual es estructuras formativas que permitan contrastar la validez y legitimidad de la propuesta resultado de la investigación, c) La Comunidad Educativa En General: 1- Es necesario insistir que la formación Técnica Profesional y Tecnológica forman parte del Sistema de Educación Superior en Colombia que persigue dar respuesta a necesidades de formación en estos niveles, razón por la cual, es conveniente evitar la valoración y discriminación que usualmente se hace, dado que todo sistema, entendido como el conjunto de elementos 
que interactúan entre si orientados a la consecución de un mismo propósito, tienen metas y tareas diferentes, que no pueden ser utilizadas como razones de exclusión y valoración negativa, sino por el contrario, deben ser asumidas como opciones de calidad, opor tunidad y formación concretas. 2- Los resultados de la investigación, corroboran la seriedad y rigor con las cuales las diferentes instituciones de educación superior vienen asumiendo la formación Técnica Profesional y Tecnológica y se convierten en oportunidades claras, viables y pertinentes para consolidar su formación como opción concreta de desarrollo profesional.

El proceso desarrollado en la investigación efectuada no se agota con la presentación de la misma, sino por el contrario es necesario considerarlo como un aporte o una contribución a la compleja realidad de la Educación Superior y especialmente de la formación Técnica Profesional yTecnológica, esperando sea aporte a futuros procesos investigativos en los cuales la educación sea el eje central.

Agradecimiento: El autor agradece a la Corporación Tecnológica de Bogotá, por su apoyo institucional y a todas aquellas personas e instituciones que de alguna manera colaboraron para llevar a feliz término el desarrollo de la investigación base del presente artículo.

\section{Referentes Bibliográficos}

1. ARIAS OSORIO, Eduardo. El potencial humano de alto nivel en Colombia. Santa Fe de Bogotá, D.C.r Mineducación, ACUP, ICETEX, 1ª Edición 1998, p. 1ó2.

2. DÍAZ, M. y LÓPEZ, J. "Formación de profesionales a partir de estructuras curriculares sustentadas en investigación", elaborado para el CNA. Abril de 1999.

3. FERNANDEZGUERREO, Marta M, GÓMEZ PAZ, Mireya, SANCHEZ GÓMEZ, Luz Marina, CHICO RUIZ, Mar co Antonio. La enseñanza diferenciada, una estrategia de calidad en la Fundación Tecnológica Antonio de Arévalo, TECNAR. Ediciones Pluma de Mompox S.A, Cartagena de Indias, 2008.

4. FERNANDEZ GUERREO, Marta Mr, GÓMEZ PAZ, Mireya, CHICO RUIZ, Marco Antonio, MACÍAS GÓMEZ, María Escolástica, Formación del Profesor para una Enseñanza Diferenciada, una respuesta a las nuevas tendencias de la educación superior. Ediciones TECNAR, Cartagena de Indias, 2010.

5. GARDNER, Howard, Inteligencias múltiples: La teoría de las inteligencias múltiples, Editorial Paidós, 1995. 
6. GÓMEZ BUENDÍA, Hernando. Educación para la ciencia y la tecnología. En: Educación: La agenda del siglo XXI. Santa Fe de Bogotá, D.C., PNUD Tercer Mundo Editores, 1999, p. 316.

7. GÓMEZ, Víctor Manuel. La formación técnica y tecnológica en el ámbito internacional, 1990

8. GÓMEZ Campo, Víctor Manuel. La educación tecnológica en la Visión Colombia 2019, en revista Javeriana, ISSN 0120-3088, No.733,2007, págs. 78-82

9. GÓMEZ Campo, Víctor Manuel. DIAZ RIOS, Claudia Milena. CELIS GIRALDO, Jorge Enrique. El puente está quebrado. Aportes a la reconstrucción de la educación media en Colombia. Editorial Universidad Nacional, Bogotá, Colombia, enero 2009.

10. GOMEZ CAMPO, Víctor Manuel, La educación tecnológica en Colombia, Editorial Universidad Nacional, Bogotá, Colombia, 1995.

11. GOMEZ CAMPO, Víctor Manuel, Cuatro temas críticos de la educación superior en Colombia: Estado, Instituciones, pertinencia y Equidad Social, Alfa omega S.A., Colombia, octubre de 2000.

12. HOYOS REGINO, Santander Enrique, y otros, Currículo y Planeación Educativa, Cooperativa Editorial Magisterio, Bogotá, D.C. Colombia 2004

13. LÓPEZ JMMÉNEZ, Nelson Ernesto, PUENTES DE VELÁSQUEZ Ana Victoria, Evaluación de la calidad de la educación en Colombia, Estado del Arte, Segunda fase, grupo PACA, Universidad Surcolombiana, Neiva, noviembre de 2011

14. LÓPEZ JMMÉNEZ, Nelson Ernesto, PUENTES DE VELÁSQUEZ Ana Victoria y otros, Estado del Arte: Evaluación de la calidad de la educación en Colombia, grupo PACA, Universidad Surcolombiana, Neiva, mayo de 2007

15. LÓPEZ JIMÉNEZ, Nelson Ernesto, Evaluación por competencias: Un reto por enfrentar y un proceso por construir, Grupo de Investigación P.AC.A, Universidad Surcolombiana, Litocentral Ltda., Neiva, Huila, 2006.

16. La de-construcción curricular, Colección Seminarium MAGISTERIO, Línea de investigación Curriculum, Cooperativa Editorial Magisterio, 2001 ..

17. La reestructuración curricular de la educación superior, Hacia la integración del saber. ICFES, Bogotá, 1995. 
18. - Modelos y Prácticas pedagógicas en la Educación Superior. Grupo de investigación PACA, Universidad Surcolombiana, Editora Guadalupe Ltda. Abril de 2004

19. LÓPEZ JIMÉNEZ, Nelson Ernesto, PUENTES DE VELÁSQUEZ Ana Victoria, Modernización Curricular de la Universidad Surcolombiana. Grupo de investigación PACA, Universidad Surcolombiana, Litocentral editores S.A.S. Noviembre de 2011 .

20. MARTIN BARBERO, Jesús. "Saberes hoy: diseminaciones, competencias y transversalidades, Revista lberoamericana de Educación. No 32. 2003

21. Ministerio de Educación Nacional, Balance del Plan Nacional de Educación 1996-2006, La educación un compromiso de todos, CEDE, Bogotá, agosto de 2006.

22. - Educación de calidad, El camino para la prosperidad, Bogotá, Colombia Noviembre de 2010

23. Educación Técnica Y Tecnológica para la Competitividad, SIAG LTDA, Bogotá, 2008,

24. gotá, Colombia, enero de 2008. Revolución Educativa: Plan sectorial 2006-2010, Bo-

25. Misión de Ciencia, Educación y Desarrollo. Colombia al filo de la oportunidad. Santa Fe de Bogotá, Colección Mesa Redonda, Cooperativa Editorial Magisterio, 1995, p.55.

2ó. MEJía Marco Raúl. Educación y Escuela en el Fin de Siglo. Bogotá, CINEP, 1995.

27. MEN, propuesta para el diseño y evaluación de Programas Técnicos y Tecnológicos y por Ciclos propedéuticos

28. PANESSO TASCON, Jairo, La Educación Tecnológica en Colombia, problemática y perspectiva. Colección Treinta Años, Instituto Tecnológico Municipal Antonio José Camacho, Talleres Gráficos de Litociencia, Santiago de Cali, febrero de 2000 .

29. PETROSINO, Jorge. La educación tecnológica: ¿Un intento de renovar la educación técnica? 
30. TECNAR, Fundación Tecnológica Antonio de Arévalo, La educación Técnica Profesional y Tecnológica, una experiencia institucional, Cartagena de Indias, Colombia, 2007.

31. TÜNNERMANN B. Carlos. Aproximación histórica a la universidad y su problemática actual. ICFES-Uniandes. Santa Fe de Bogotá, D.C., Siglo XXI Editores, 1997, p. 99.

32. http://www.cna.gov.co/1741/article-187279.html

33. http://www.dnp.gov.co/PND/PND20102014.aspx

34. http://www.mineducacion,gov.co/1621/articles-85888_archivo_pdf.pdf

35. www.mineducacion.gov.co/1621/article-85906.html

36. http://www.mineducacion.gov.co/1ó21/articles-93ó47_archivo_pdf_plansectorial.pdf

37. http://menweb.mineducacion,gov.co/info_sector/estadisticas/superior/index.html

38. http://www.mineducacion.gov.co/sistemasdeinf ormacion/1735/artides-212350_ resumenxis

39. http://200.41.9227:7777/men/consultas/ContenedorRepConsultas.jsp

40. http://www.mineducacion.gov.co/1ó21/articles293647_archivo_pdf_ plansectorial.pdf

41. http://menweb.mineducacion,gov.co/info_sector/estadisticas/index.html

42. hthttp://menweb.mineducacion.gov.co/info_sector/estadisticas/superior/ matricula_nivel.html

43. http://menweb.mineducacion.gov.co/info_sector/estadisticas/superior/ matricula_nivel_sexo.htm!

44. http://www.slideshare.net/guest975e5ó/educacion-tecnica-y-tecnologia-en-colombia-ok

45. http://www.slideshare.net/MRDIXON/sistema-de-educacion 


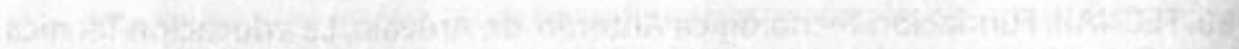

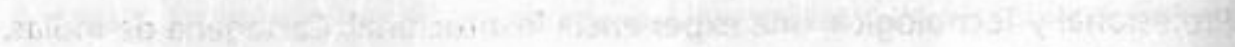

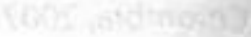

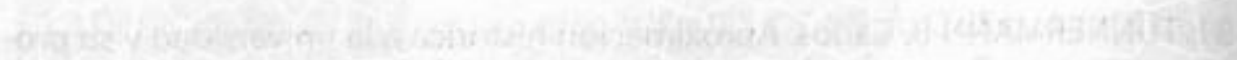

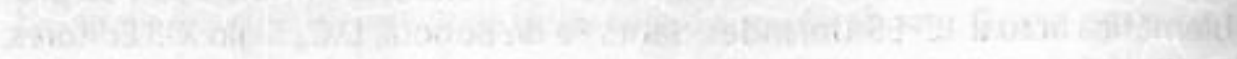

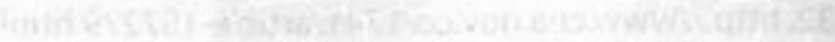

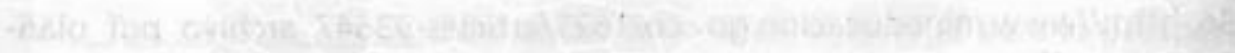

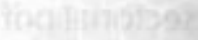

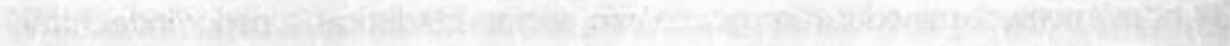

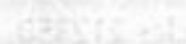

$x_{n+2}$

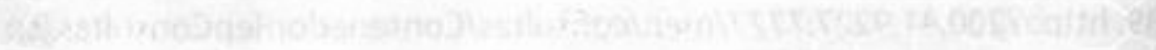

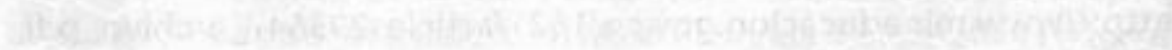

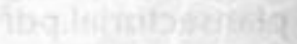

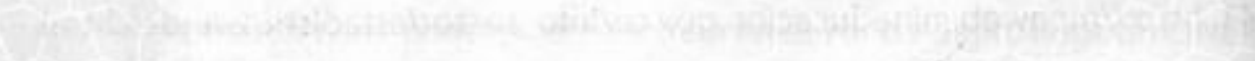

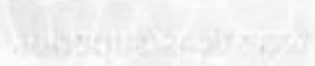

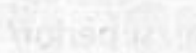

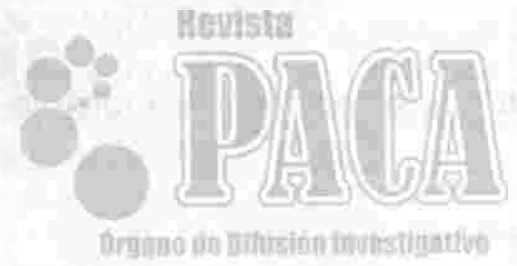

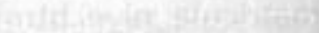

ungino of 